\title{
Characterization of a prognostic four-gene methylation signature associated with radiotherapy for head and neck squamous cell carcinoma
}

\author{
JIABAO MA, RUI LI and JIE WANG \\ Department of Radiation Oncology, Sichuan Cancer Hospital, Chengdu, Sichuan 610041, P.R. China
}

Received September 10, 2018; Accepted March 15, 2019

DOI: $10.3892 / \mathrm{mmr} .2019 .10294$

\begin{abstract}
Head and neck squamous cell carcinoma (HNSCC) remains one of the most common malignancies associated with poor prognosis. DNA methylation has emerged as an important mechanism underlying the radio-resistance of tumors. Prognostic biomarkers based on radiotherapy-related aberrant DNA methylation are limited. Methylation profiles of 388 patients with HNSCC were acquired from The Cancer Genome Atlas (TCGA) portal. Genes with differentially methylated $\mathrm{CpG}$ sites (DMGs) were screened between patients with a favorable and poor prognosis with or without radiotherapy. A weight gene co-methylation network was constructed using a Weighted Gene Co-expression Network Analysis (WGCNA) package. A lasso Cox-PH model was used to identify the optimal panel of genes with the ability to predict survival in these patients. Prognostic performance of the multi-gene methylation signature was assessed in a training set and confirmed in a validation set. A total of 976 DMGs were observed between favorable and poor prognostic samples. Four DMG-enriched co-methylation modules were identified. A four-gene methylation signature was determined by the lasso Cox-PH model that consisted of ZNF10, TMPRSS12, ERGIC2, and $R N F 215$. The risk score based on the four-gene signature was able to divide the training or validation set into two risk groups with significantly different overall survival. Thus, the present study revealed a radiotherapy-related four-gene methylation signature to predict survival outcomes of patients with
\end{abstract}

Correspondence to: Dr Jie Wang, Department of Radiation Oncology, Sichuan Cancer Hospital, 55, Section 4 of South Renmin Road, Chengdu, Sichuan 610041, P.R. China

E-mail: wangjie201208@sina.com

Abbreviations: HNSCC, head and neck squamous cell carcinoma; TCGA, The Cancer Genome Atlas; DMG, differentially methylated CpG site; WGCNA, Weighted Gene Co-expression Network Analysis; HPV, human papilloma virus; OS, overall survival; FDR, false discovery rate; FC, fold change

Key words: HNSCC, DNA methylation, prognostic signature, radiotherapy, WGCNA
HNSCC, providing candidate therapeutic targets for novel therapy against HNSCC. However, substantial validation experiments are required.

\section{Introduction}

Head and neck squamous cell carcinoma (HNSCC) refers to a group of malignancies that originates in the oral cavity, oropharynx, larynx, or hypopharynx $(1,2)$. It is the seventh most common cancer worldwide with an annual incidence of more than half a million (3). The average 5-year overall survival (OS) of patients with HNSCC is between 42-64\% (4). Given the intrinsic heterogeneity of this disease, the identification of prognostic gene signatures is of a particular interest to improve HNSCC diagnosis.

DNA methylation patterns are largely altered in cancer cells as compared with normal cells (5). Epigenetic gene silencing caused by DNA methylation has been widely accepted as an important mechanism of tumorigenesis (6). Studies have revealed the aberrant methylation of multiple genes in HNSCC $(7,8)$. Kostareli et al demonstrated and confirmed a human papilloma virus (HPV)-related prognostic methylation score for patients with HNSCC $(9,10)$. Moreover, a recent study identified an HPV infection-related epigenetic signature consisting of five $\mathrm{CpGs}$ as survival predictors in HPV-positive HNSCC (11). Despite these marked findings, there is a lack of a reliable methylation prognostic signature for risk stratification in patients with HNSCC.

Similar to surgery, radiotherapy alone may be used for HNSCC treatment at early stages. For patients with HNSCC in middle and late stages, radiotherapy is generally implemented in combination with surgical excision that may decrease local recurrence and improve tumor control and the survival of patients (12). DNA methylation is known to play a critical role in the resistance of tumors to radiotherapy (13). Epigenetic silencing of tumor suppressor genes by methylation was revealed to be associated with radio-resistance of oral squamous cell carcinoma and poor outcome of patients (14). Given the close associations between DNA methylation and radiotherapy, herein we focused on the identification of a prognostic methylation signature from radiotherapy-related differentially methylated CpG sites based on the methylation data obtained from 388 patients with HNSCC from The Cancer Genome Atlas (TCGA) database. The prognostic robustness of this 
methylation signature was assessed in a training set as well as a validation set.

\section{Materials and methods}

Retrieval of public data. The DNA methylation profile of 580 patients with HNSCC was retrieved from TCGA data portal (https://tcga-data.nci.nih.gov/tcga/) based on the Illumina Infinium Human Methylation 450 BeadChip platform. Of these patients, 388 with corresponding clinical data concerning radiotherapy and survival were selected as a training cohort. The GSE75537 (15) dataset downloaded from the National Center for Biotechnology Information Gene Expression Omnibus repositories (http://www.ncbi.nlm.nih. gov/geo/) contained 108 samples of oral tongue squamous cell carcinomas, and 53 of these samples with available survival information were selected as a validation cohort.

Differential methylation analyses between favorable and poor prognostic samples. Poor prognostic samples (patients not receiving radiotherapy with an OS of 12 months or shorter) and favorable prognostic samples (patients receiving radiotherapy with an OS of 48 months or longer) were selected from the training cohort. The acquired methylated sites were annotated based on the platform annotation information, and only those in the $\mathrm{CpG}$ sites were retained. The genes with differentially methylated $\mathrm{CpG}$ sites (DMGs) between the two groups were selected using the limma package (16) (version 3.34.7, https://bioconductor. org/packages/release/bioc/html/limma.html) with significant cut-off values of false discovery rate $($ FDR $)<0.05$ and $\log _{2}$ fold change $(\mathrm{FC}) \mid>0.1$.

Selection of co-methylation modules. A weighted gene co-methylation network with all methylated CpGs obtained from the training set was constructed using the Weighted Gene Co-expression Network Analysis (WGCNA) software $(17,18)$ (https://cran.r-project.org/web/packages/WGCNA/index.html). To achieve scale-free topology, a soft-thresholding power of $\beta=5$ with a scale-free $R^{2}$ value of 0.9 for calculating adjacency, was selected. The genes with similar methylation levels were grouped into the same module. The modules with minSize $=100$ and cutHeight $=0.95$ were identified by dynamic tree cut algorithm using dynamicTreeCut version 1.63 (https://cran.r-project. org/web/packages/dynamicTreeCut/index.html). Enrichment analysis of DMGs was carried out in each identified module with a hypergeometric-based test (19). The modules with $\mathrm{P}<0.05$ and fold enrichment $>1$ were selected as DMG-enriched modules, which were subjected to gene ontology (GO) (20) functional enrichment analysis using the DAVID (https://david. ncifcrf.gov/) bioinformatics online tool (21).

Correlation analysis of methylation and expression data. Methylation and expression data of the DMGs were focused on in these selected DMG-enriched modules. Using methylation data and the matched mRNA expression data, overall methylation levels of all DMGs and their overall gene expression levels were correlated by calculating Pearson correlation coefficient (CC) using the cor.test function (https://stat.ethz.ch/R-manual/ R-devel/library/stats/html/cor.test.html). Correlation between
Table I. Clinical covariates of patients in the training set and the validation set.

\begin{tabular}{lcc}
\hline $\begin{array}{l}\text { Clinical } \\
\text { covariates }\end{array}$ & $\begin{array}{c}\text { Training } \\
\text { set }(\mathrm{n}=388)\end{array}$ & $\begin{array}{c}\text { Validation } \\
\text { set }(\mathrm{n}=53)\end{array}$ \\
\hline Age (mean $\pm \mathrm{SD}$, years) & $60.81 \pm 11.66$ & $49.36 \pm 13.47$ \\
Sex (male/female) & $288 / 100$ & $42 / 11$ \\
Death (dead/alive/-) & $117 / 271$ & $15 / 38$ \\
OS time (mean \pm SD, months) & $26.43 \pm 26.21$ & $30.46 \pm 26.98$ \\
\hline
\end{tabular}

OS, overall survival; $\mathrm{SD}$, standard deviation.

methylation levels and expression levels was explored for every individual DMG. As a result, the genes with a negative $\mathrm{CC}$ value were selected for further analysis.

Statistical analysis for survival. Based on the survival information of patients in the training set, a univariate Cox regression analysis was performed to evaluate the association of the aforementioned genes which had a negative $\mathrm{CC}$ with prognosis. The significant genes with a log-rank $\mathrm{P}<0.05$ were regarded as prognosis-related genes, which were used as input for a L1 penalized (LASSO) Cox-proportional hazard (PH) model (22) to identify the optimal panel of prognostic genes using the penalized package (https://cran.r-project.org/web/packages/penalized/) of $\mathrm{R}$ language (version 3.4.1). Cox-PH coefficients and methylation levels of these prognostic genes were combined to construct the following prognostic model:

$$
\text { Risk score }=\sum \text { coef }_{\text {gene }} \times \text { Methylation }_{\text {gene }}
$$

where, Coef ${ }_{\text {gene }}$ represents the Cox-PH coefficient of an indi-

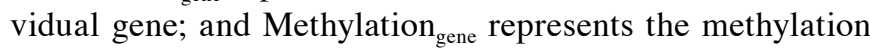
level of an individual gene.

A risk score was assigned to each sample in the training cohort. With a median methylation risk score as the cutoff, the training set was dichotomized into a high-risk group and a low-risk group. Survival probabilities of the two groups were analyzed by Kaplan-Meier estimates (23) using survival package of $\mathrm{R}$ language. P-values from the log-rank test suggested significance of the prognostic model. Specificity and sensitivity of this model were assessed by the area under the receiver operating characteristic (ROC) curve (AUC) analysis. Prognostic performance of the methylation signature was assessed in the validation cohort.

\section{Results}

Identification of radiotherapy-related DMGs. Clinical and demographic data of the training and validation cohorts are presented in Table I. Based on the aforementioned criterion of sample classification, the training cohort had 30 poor prognostic samples and 27 favorable prognostic samples. Between the poor and favorable prognostic samples, a total of 976 DMGs associated with radiotherapy $\left(3.43 \times 10^{-8}<\right.$ Pnominal $<6.807 \times 10^{-4}$, FDR $\left.<0.05\right)$ were obtained by differential methylation analysis (Fig. 1A). Among these DMGs, 476 (48.77\%) 

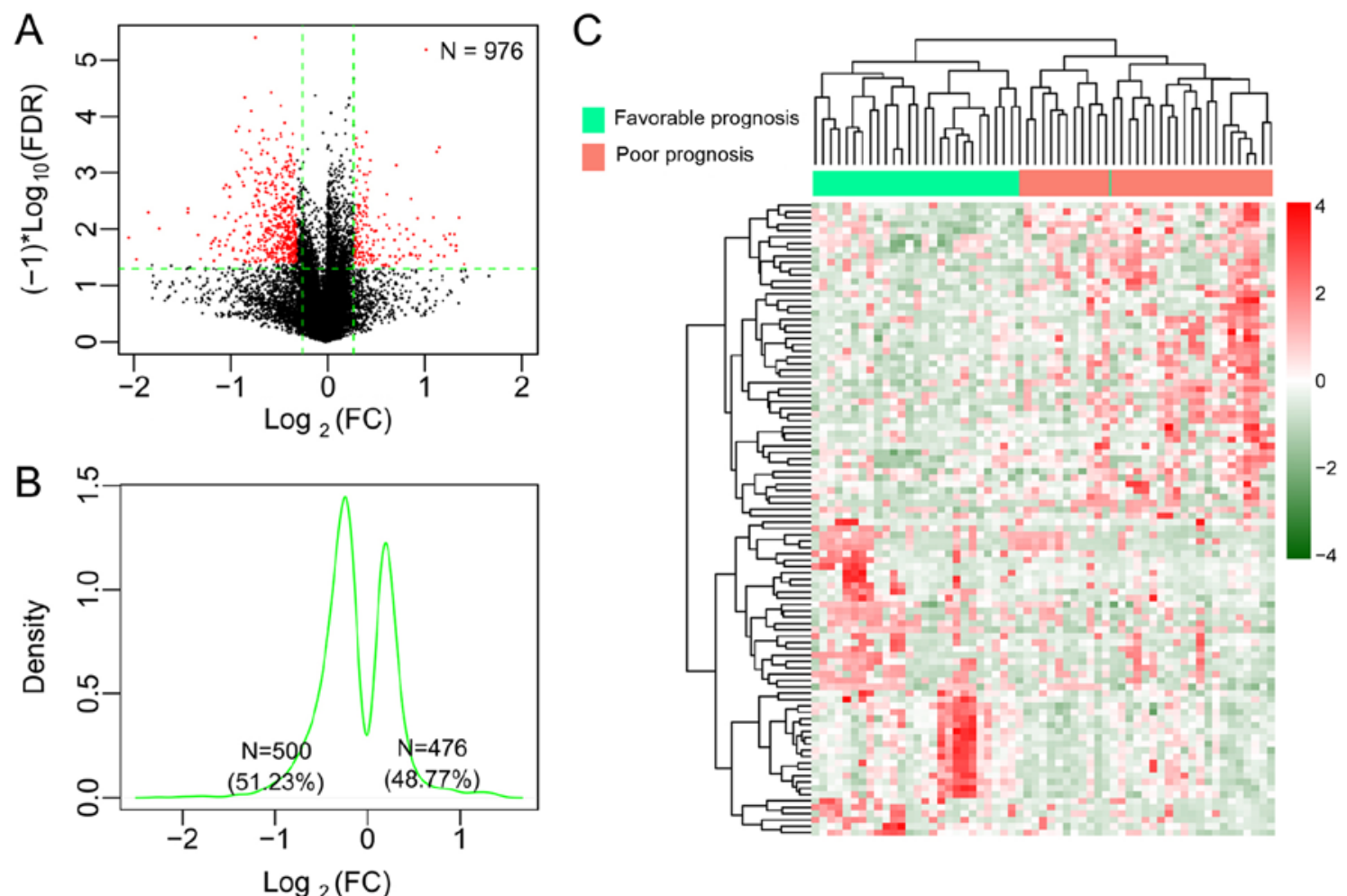

Figure 1. Analysis of DMGs between favorable and poor prognostic samples of the training set. (A) Volcano plot of effect size ( $\log _{2}[$ fold changes]) against $-\log _{10}(\mathrm{FDR})$ of DMGs. The red spots stand for DMGs with FDR $<0.05$ and $\log _{2} \mathrm{FCl}>0.1$, while black spots stand for non-significant DMGs. The horizontal green dash line denotes FDR $<0.05$; the vertical green dash lines denote $\log _{2} \mathrm{FCl}>0.1$. (B) Kernel density plot of $\log _{2}$ (fold changes) of DMGs. (C) Two-way hierarchical clustering analysis of favorable and poor prognostic samples based on methylation levels of the top 100 DMGs. Color mapping from green to red indicates methylation level from low to high. DMGs, differentially methylated CpG sites; FDR, false discovery rate.

were hypermethylated and 500, hypomethylated (Fig. 1B). The two-way hierarchical clustering analysis based on methylation levels of the top 100 DMGs revealed the difference in the DNA methylation pattern between the favorable prognostic samples and the poor prognostic samples (Fig. 1C). With regard to the CpG sites in the 976 DMGs, 192 DMGs were located in the TSS region, 220 in the gene body region, 30 in the first exon, 53 in the 5'-untranslated regions (UTRs), 35 in the 3'-UTRs, and 446 in the promoters. All significant DMGs were ranked according to the FDR value. As a consequence, the top 20 DMGs with significance were selected (Table II).

WGCNA network analysis and key module identification. To evaluate the correlation between all methylated CpGs obtained from the training dataset, a weighted gene co-methylation network was applied to these methylated CpGs. Twelve modules of co-methylated genes were identified, wherein $\mathrm{CC}$ varied from 0.514 to 0.772 (mean value $=0.642$, Table III and Fig. 2A). Other CpGs that exhibited no significant correlation with methylation levels were grouped into a grey module. The enrichment of DMGs was evaluated in these modules. As revealed in Fig. 2B and C, yellow-green, magenta, purple, and turquoise modules had 44, 92, 45 and 113 DMGs, respectively, and each of these met the criteria of fold enrichment $>1$ and $\mathrm{P}<0.05$. Thus, the four DMG-enriched modules were subjected to GO enrichment analysis. All DMGs in the four modules were predominately linked to protein-DNA complex assembly, nucleosome organization, chromatin assembly, and the cell cycle process (Table IV).
Identification and validation of a four-gene prognostic methylation signature. In correlation analysis, an inverse correlation was evident between overall methylation and overall expression of all DMGs in the four DMG-enriched modules ( $\mathrm{CC}=-0.5051, \mathrm{P}=2.20 \times 10^{-16}$; Fig. 3 ). In addition, an inverse correlation was also observed between methylation and gene expression of individual genes for 165 genes. Univariate Cox regression analysis revealed that 34 genes were significantly related to prognosis in methylation levels, 41 genes had a significant association with prognosis in gene expression levels, and 10 were overlapped (Fig. 4).

A lasso Cox-PH model was fit using the overlapped 10 genes to identify the optimal panel of methylation genes for prognosis prediction. The parameter $\lambda$ value was tuned to 0.1845 by conducting 1,000 simulations of cross-validation to obtain a maximal cross-validation likelihood (cvl) value of -720.354. A panel of four genes was obtained under this condition, including zinc finger protein $(Z N F) 10$, transmembrane serine protease (TMPRSS)12, endoplasmic reticulum-Golgi intermediate compartment protein $(E R G I C) 2$, and ring finger protein $(R N F) 215$.

It was investigated whether the methylation level of the four genes was associated with prognosis. As revealed in Fig. 5A, all patients from the training set were divided into hypermethylated and hypomethylated groups based on the median methylation level of each gene. A Kaplan-Meier plot revealed that the patients with hypomethylated ZNF10 had significantly better survival outcome than those with hypermethylated ZNF1O (P=0.0434). The same result 
Table II. Top 20 DMGs between favorable and poor prognostic samples.

\begin{tabular}{|c|c|c|c|c|c|c|c|c|c|}
\hline ID & Chr & Position & Gene & Location & $\beta$-favorable & $\beta$-poor & Effect & Pnominal & FDR \\
\hline $\operatorname{cg} 26934993$ & $\operatorname{chr} 17$ & 37528248 & KAT2A & TSS200 & 0.3667 & 0.6062 & -0.7253 & $5.43 \times 10^{-8}$ & $3.980 \times 10^{-6}$ \\
\hline $\operatorname{cg} 26370886$ & $\operatorname{chr} 19$ & 53930240 & RASIP1 & Body & 0.3655 & 0.5390 & -0.5604 & $5.12 \times 10^{-7}$ & $3.750 \times 10^{-5}$ \\
\hline $\operatorname{cg} 24351916$ & $\operatorname{chr} 3$ & 39427639 & RPSA & Body & 0.7753 & 0.8339 & -0.1051 & $5.82 \times 10^{-7}$ & $4.270 \times 10^{-5}$ \\
\hline cg09938490 & $\operatorname{chr} 15$ & 41876101 & SERINC4 & Body & 0.8672 & 0.7346 & 0.2394 & $8.47 \times 10^{-7}$ & $6.210 \times 10^{-5}$ \\
\hline cg08069338 & chr6 & 160127888 & SNORA29 & TSS1500 & 0.8294 & 0.7166 & 0.2110 & $9.56 \times 10^{-7}$ & $7.010 \times 10^{-5}$ \\
\hline $\operatorname{cg} 27211576$ & $\operatorname{chr} 15$ & 72880218 & CSK & Body & 0.6376 & 0.5534 & 0.2045 & $1.60 \times 10^{-6}$ & $1.171 \times 10^{-4}$ \\
\hline $\operatorname{cg} 26264697$ & chr19 & 3529064 & HMG20B & Body & 0.4428 & 0.5928 & -0.4210 & $1.78 \times 10^{-6}$ & $1.307 \times 10^{-4}$ \\
\hline $\operatorname{cg} 27266479$ & chr1 & 9217469 & H6PD & Promoter & 0.1574 & 0.1290 & 0.2868 & $1.95 \times 10^{-6}$ & $1.432 \times 10^{-4}$ \\
\hline $\operatorname{cg} 21657521$ & chr19 & 7650447 & C19orf59 & TSS1500 & 0.0907 & 0.1250 & -0.4637 & $2.75 \times 10^{-6}$ & $2.017 \times 10^{-4}$ \\
\hline $\operatorname{cg} 05657416$ & chr6 & 27213771 & HIST1H4I & TSS1500 & 0.8716 & 0.8073 & 0.1106 & $2.86 \times 10^{-6}$ & $2.099 \times 10^{-4}$ \\
\hline $\operatorname{cg} 21205305$ & chr19 & 54884437 & C19orf76 & Promoter & 0.2889 & 0.3970 & -0.4583 & $3.09 \times 10^{-6}$ & $2.263 \times 10^{-4}$ \\
\hline $\operatorname{cg} 27537591$ & $\operatorname{chr} 10$ & 116687888 & TRUB1 & Promoter & 0.0501 & 0.0402 & 0.3154 & $3.30 \times 10^{-6}$ & $2.422 \times 10^{-4}$ \\
\hline $\operatorname{cg} 25739003$ & chr11 & 62097836 & EEF1G & Promoter & 0.0751 & 0.0606 & 0.3092 & $4.23 \times 10^{-6}$ & $3.100 \times 10^{-4}$ \\
\hline $\operatorname{cg} 26740494$ & chr1 & 1556994 & MMP23B & TSS1500 & 0.4795 & 0.6275 & -0.3881 & $4.68 \times 10^{-6}$ & $3.434 \times 10^{-4}$ \\
\hline $\operatorname{cg} 26222042$ & $\operatorname{chr} 5$ & 31567957 & C5orf22 & Promoter & 0.0580 & 0.0447 & 0.3762 & $4.68 \times 10^{-6}$ & $3.434 \times 10^{-4}$ \\
\hline $\operatorname{cg} 26615259$ & chr1 & 154977769 & MRPL24 & Promoter & 0.0558 & 0.0447 & 0.3208 & $4.75 \times 10^{-6}$ & $3.483 \times 10^{-4}$ \\
\hline $\operatorname{cg} 27085584$ & $\operatorname{chr} 5$ & 61735043 & DIMT1L & Promoter & 0.0392 & 0.0496 & -0.3411 & $4.85 \times 10^{-6}$ & $3.555 \times 10^{-4}$ \\
\hline $\operatorname{cg} 27535410$ & chr19 & 797354 & PRTN3 & Body & 0.8207 & 0.9326 & -0.1844 & $5.20 \times 10^{-6}$ & $3.812 \times 10^{-4}$ \\
\hline $\operatorname{cg} 27065374$ & $\operatorname{chrX}$ & 67976906 & EFNB1 & Body & 0.5659 & 0.4863 & 0.2185 & $5.20 \times 10^{-6}$ & $3.816 \times 10^{-4}$ \\
\hline $\operatorname{cg} 22961457$ & $\operatorname{chr} 20$ & 61840754 & SLC2A4RG & 3'UTR & 0.2397 & 0.4364 & -0.8641 & $5.41 \times 10^{-6}$ & $3.965 \times 10^{-4}$ \\
\hline
\end{tabular}

$\beta$-favorable and $\beta$-poor represent the mean methylation level of favorable and poor prognostic samples, respectively. Chr, chromosome; FDR, false discovery rate; DMG, genes with differentially methylated $\mathrm{CpG}$ sites.

Table III. WGCNA network analysis identified gene modules with co-methylated CpG sites.

\begin{tabular}{lccccc}
\hline Module color & Count of CpGs & Correlation & Pcorr & Count of DM CpGs & Enrichment fold (95\% CI) \\
\hline Black & 301 & 0.702 & $7.01 \times 10^{-32}$ & 30 & $1.033(0.679-1.521)$ \\
Blue & 562 & 0.514 & $1.70 \times 10^{-6}$ & 38 & $0.701(0.486-0.985)$ \\
Brown & 469 & 0.689 & $6.68 \times 10^{-11}$ & 28 & $0.619(0.403-0.915)$ \\
Green & 397 & 0.561 & $1.33 \times 10^{-7}$ & 46 & $1.201(0.856-1.652)$ \\
Green-yellow & 196 & 0.687 & $2.14 \times 10^{-33}$ & 44 & $2.327(1.622-3.277)$ \\
Grey & 2,623 & 0.215 & $8.56 \times 10^{-2}$ & 180 & $0.711(0.596-0.846)$ \\
Magenta & 221 & 0.642 & $7.05 \times 10^{-10}$ & 92 & $4.314(3.301-5.604)$ \\
Pink & 248 & 0.565 & $1.59 \times 10^{-4}$ & 2 & $0.084(0.010-0.306)$ \\
Purple & 208 & 0.687 & $2.71 \times 10^{-23}$ & 45 & $2.20 \times 10^{-6}$ \\
Red & 324 & 0.701 & $5.87 \times 10^{-4}$ & 13 & $9.75 \times 10^{-8}$ \\
Tan & 182 & 0.772 & $4.53 \times 10^{-26}$ & 16 & $0.416(0.218-0.727)$ \\
Turquoise & 649 & 0.645 & $1.47 \times 10^{-27}$ & 113 & $0.911(0.507-1.532)$ \\
Yellow & 431 & 0.538 & $1.66 \times 10^{-15}$ & 10 & $1.805(1.442-2.245)$ \\
\hline
\end{tabular}

WGCNA, Weighted Gene Co-expression Network Analysis; Count of CpGs, the number of CpGs in a module; Count of DM CpGs, the number of differentially methylated CpGs enriched in a module; Pcorr, P-value for correlation coefficient; Phyper, P-value for enrichment analysis.

was observed for TMPRSS12 $(\mathrm{P}=0.0386)$ and ERGIC2 $\left(\mathrm{P}=3.093 \times 10^{-4}\right)$. Conversely, a significantly worse prognosis was observed in the patients with hypomethylated $R N F 215$ as compared to those with hypermethylated $R N F 215$ $(\mathrm{P}=0.0129)$.
The relationship between gene expression of each of the four genes and survival was explored. Based on the median gene expression level, all samples in the training set were classified into high expression and low expression groups. ZNF10, TMPRSS12 and ERGIC2 were associated with significantly 


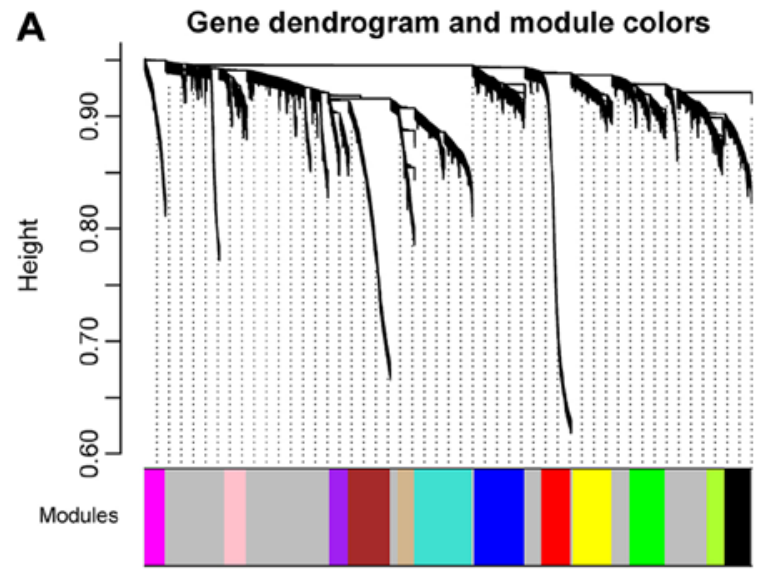

B
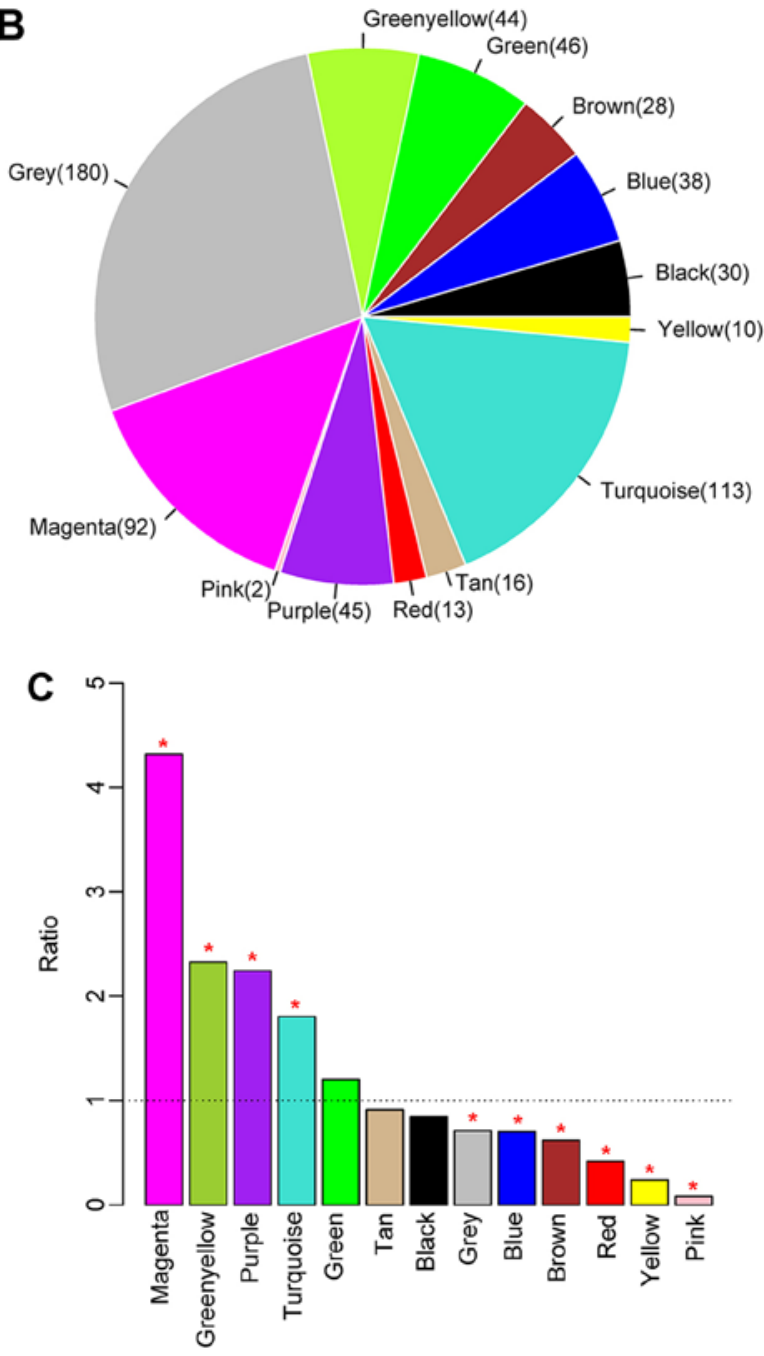

Figure 2. Results of the WGCNA. (A) Clustering dendrograms of gene modules associated with co-methylated $\mathrm{CpG}$ sites. Genes in the same branch were highly connected. Each color indicates a certain gene. (B) The count of significant DMGs mapped in each gene module. Each color represents a gene module, and the number represents the genes with differentially methylated CpGs. (C) Fold-enrichment value of each module. The vertical axis stands for the fold enrichment value. The horizontal black dash line denotes fold enrichment $=1$. ${ }^{*} \mathrm{P}<0.05$. WGCNA, Weighted Gene Co-expression Network Analysis; DMGs, differentially methylated CpG sites.

longer $\mathrm{OS}$ in the high expression group than in the low expression group $\left(\mathrm{P}=1.125 \times 10^{-4}, \mathrm{P}=0.0471, \mathrm{P}=1.196 \times 10^{-3}\right.$; Fig. $\left.5 \mathrm{~B}\right)$. An opposite result was observed for RNF215; patients with

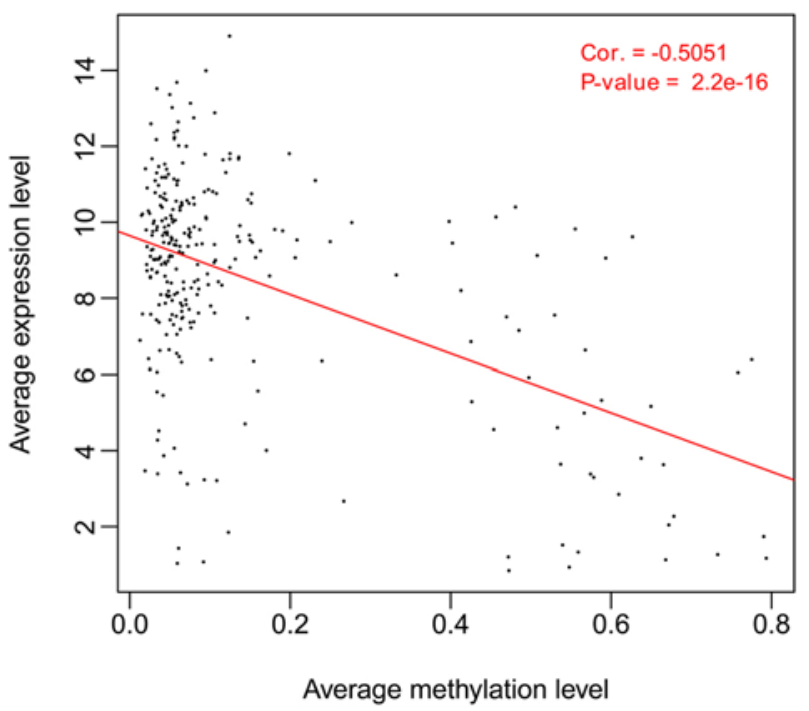

Figure 3. Scatter plot of the overall correlation between methylation and gene expression levels of the paired samples in TCGA portal for the 294 genes contained in the significantly enriched four gene modules, yellow-green, magenta, purple, and turquoise modules. The black spots represent genes. The red line is the trend line of the genes. Cor. denotes Pearson's correlation coefficient of methylation and gene expression. The P-value indicates the significance of the correlation.

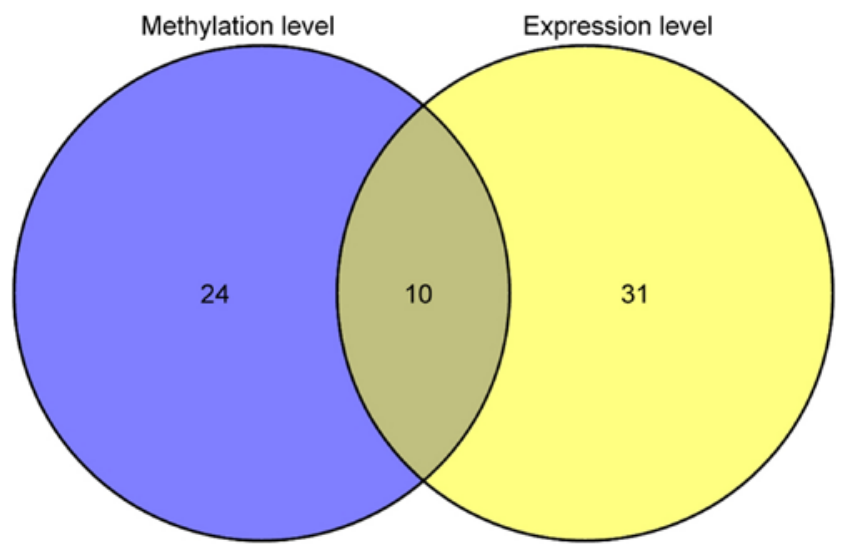

Figure 4. A Venn diagram depicting overlap between the genes significantly related to prognosis in methylation level (left) and the genes significantly associated with prognosis in gene expression (right). Two groups of genes were identified by univariate Cox regression analysis.

high $R N F 215$ expression had significantly shorter OS than those with low expression ( $\mathrm{P}=0.0391$; Fig. 5B).

A risk prediction model was constructed that included risk score based on the Cox-PH prognostic correlation of the optimal four-gene panel (Table V):

Risk score $=(0.9639) \times$ Methylation ${ }_{\mathrm{cg} 25577680}+(1.035) \mathrm{x}$ Methylation $_{\mathrm{cg} 27261219}+(7.1664) \mathrm{x}$ Methylation ${ }_{\mathrm{cg} 25338581}+(-4.8961)$ x Methylation ${ }_{\mathrm{c} 252564984}$

The risk score was calculated for each patient. The training cohort was grouped based on the risk score into high-risk and low-risk groups. Samples in the low-risk group exhibited a significantly longer OS than those from the high-risk group $\left(\mathrm{P}=7.056 \times 10^{-3}\right.$; Fig. 6A). The AUC value of the methylation risk score was 0.957 , suggestive of the high accuracy of the four-gene methylation signature in predicting the survival of 
Table IV. Significantly enriched GO terms for genes with differentially methylated CpGs in four important gene modules.

\begin{tabular}{|c|c|c|c|}
\hline GO term & Count of genes & P-value & Genes \\
\hline Protein-DNA complex assembly & 8 & $2.71 \times 10^{-4}$ & $\begin{array}{l}\text { HIST2H2AA3, HIST4H4, HIST1H2AG, HIST1H2BL, CENPA, } \\
\text { HIST1H2BG, HIST1H3A, HIST1H2AH, MIS12 }\end{array}$ \\
\hline Nucleosome organization & 8 & $3.10 \times 10^{-4}$ & $\begin{array}{l}\text { HIST2H2AA3, HIST4H4, HIST1H2AG, HIST1H2BL, CENPA, } \\
\text { HIST1H2BG, HIST1H3A, SUPT16H, HIST1H2AH }\end{array}$ \\
\hline Protein folding & 10 & $8.25 \times 10^{-4}$ & $\begin{array}{l}\text { GRPEL1, CRYAA, PFDN5, CCT8, C19ORF2, CCT3, CCT6A, } \\
\text { DNAJC2, CLPX, PIN1 }\end{array}$ \\
\hline Nucleosome assembly & 7 & $1.10 \times 10^{-3}$ & $\begin{array}{l}\text { HIST2H2AA3, HIST4H4, HIST1H2AG, HIST1H2BL, CENPA, } \\
\text { HIST1H2BG, HIST1H3A, HIST1H2AH }\end{array}$ \\
\hline DNA packaging & 8 & $1.23 \times 10^{-3}$ & $\begin{array}{l}\text { HIST2H2AA3, CHMP1A, HIST4H4, HIST1H2AG, } \\
\text { HIST1H2BL, CENPA, HIST1H2BG, HIST1H3A, HIST1H2AH }\end{array}$ \\
\hline Chromatin assembly & 7 & $1.32 \times 10^{-3}$ & $\begin{array}{l}\text { HIST2H2AA3, HIST4H4, HIST1H2AG, HIST1H2BL, CENPA, } \\
\text { HIST1H2BG, HIST1H3A, HIST1H2AH }\end{array}$ \\
\hline $\begin{array}{l}\text { Chromatin assembly or } \\
\text { disassembly }\end{array}$ & 8 & $1.97 \times 10^{-3}$ & $\begin{array}{l}\text { HIST2H2AA3, HIST4H4, HIST1H2AG, HIST1H2BL, CENPA, } \\
\text { HIST1H2BG, HIST1H3A, SUPT16H, HIST1H2AH }\end{array}$ \\
\hline Translation & 13 & $2.31 \times 10^{-3}$ & $\begin{array}{l}\text { MRPL24, EIF4G1, RPSA, MRPS16, MRPL27, RARS, EIF2S2, } \\
\text { RPL35, MARS2, EIF5A, DPH1, RPL10A, MRPL34 }\end{array}$ \\
\hline Chromosome organization & 16 & $3.19 \times 10^{-3}$ & $\begin{array}{l}\text { KAT2A, HIST2H2AA3, HIST4H4, HIST1H2AG, HIST1H2BG, } \\
\text { NDC80, LIG4, MIS12, C20ORF20, KDM1A, CHMP1A, } \\
\text { HIST1H2BL, CENPA, HIST1H3A, SUPT16H, BRE, } \\
\text { HIST1H2AH }\end{array}$ \\
\hline Cell cycle & 21 & $5.67 \times 10^{-3}$ & $\begin{array}{l}\text { CCNT2, MAD1L1, CRYAA, NDC80, PMF1, PBK, LIG4, } \\
\text { TACC3, ESCO2, UHMK1, MIS12, PIN1, CHMP1A, PSMB6, } \\
\text { CENPA, PSMA3, SKA2, RAD51L3, MAPK7, KPNA2, } \\
\text { DNAJC2 }\end{array}$ \\
\hline DNA metabolic process & 15 & $1.11 \times 10^{-2}$ & $\begin{array}{l}\text { NEIL3, SMC5, PPT1, LIG4, ESCO2, PCNA, SUPT16H, PSIP1, } \\
\text { BRE, DDB2, RAD51L3, KPNA2, DNAJC2, APEX1, DUT }\end{array}$ \\
\hline Cell cycle process & 16 & $1.24 \times 10^{-2}$ & $\begin{array}{l}\text { MAD1L1, CRYAA, NDC80, PMF1, PBK, TACC3, UHMK1, } \\
\text { MIS12, CHMP1A, PSMB6, CENPA, PSMA3, SKA2, } \\
\text { RAD51L3, KPNA2, DNAJC2 }\end{array}$ \\
\hline Mitotic cell cycle & 12 & $1.44 \times 10^{-2}$ & $\begin{array}{l}\text { MAD1L1, CHMP1A, PSMB6, CENPA, PSMA3, NDC80, } \\
\text { SKA2, PMF1, PBK, DNAJC2, KPNA2, MIS12 }\end{array}$ \\
\hline $\begin{array}{l}\text { Response to DNA damage } \\
\text { stimulus }\end{array}$ & 12 & $1.52 \times 10^{-2}$ & $\begin{array}{l}\text { NEIL3, DDB2, BRE, SUPT16H, SMC5, PCNA, AATF, } \\
\text { RAD51L3, LIG4, ATMIN, APEX1, ESCO2 }\end{array}$ \\
\hline Chromatin organization & 12 & $1.67 \times 10^{-2}$ & $\begin{array}{l}\text { KAT2A, KDM1A, HIST2H2AA3, HIST4H4, HIST1H2AG, } \\
\text { HIST1H2BL, CENPA, HIST1H2BG, HIST1H3A, BRE, } \\
\text { SUPT16H, HIST1H2AH, C20ORF20 }\end{array}$ \\
\hline M phase & 11 & $1.68 \times 10^{-2}$ & $\begin{array}{l}\text { MAD1L1, CHMP1A, CRYAA, NDC80, SKA2, RAD51L3, } \\
\text { PMF1, PBK, TACC3, KPNA2, MIS12 }\end{array}$ \\
\hline
\end{tabular}

patients with HNSCC (Fig. 6A). The risk stratification ability of the four-gene methylation signature in the validation cohort was assessed. The validation cohort was classified based on the methylation risk score into high-risk and low-risk groups. The samples in the low-risk group also had markedly longer OS than those from the high-risk group $(\mathrm{P}=0.0145$; Fig. 6B) with an AUC value of 0.905 .

Independence of the four-gene prognostic methylation signature from radiotherapy. To investigate whether the prognostic performance of this four-gene methylation signature was independent of radiotherapy, the methylation risk score was applied to patients with or without radiotherapy. In particular, the patients without radiotherapy were classified based on the methylation risk score into high-risk and low-risk groups, and the OS of patients in the low-risk group was longer than that of patients from the high-risk group $\left(\mathrm{P}=3.632 \times 10^{-4}\right.$; Fig. 7A). The patients receiving radiotherapy were also separated as per the methylation risk score into two risk groups, and those from the low-risk group had a significantly longer OS than the patients from the high-risk group ( $\mathrm{P}=0.0281$; Fig. 7B). These results demonstrated that the prognostic value of this four-gene methylation signature was independent of radiotherapy. 

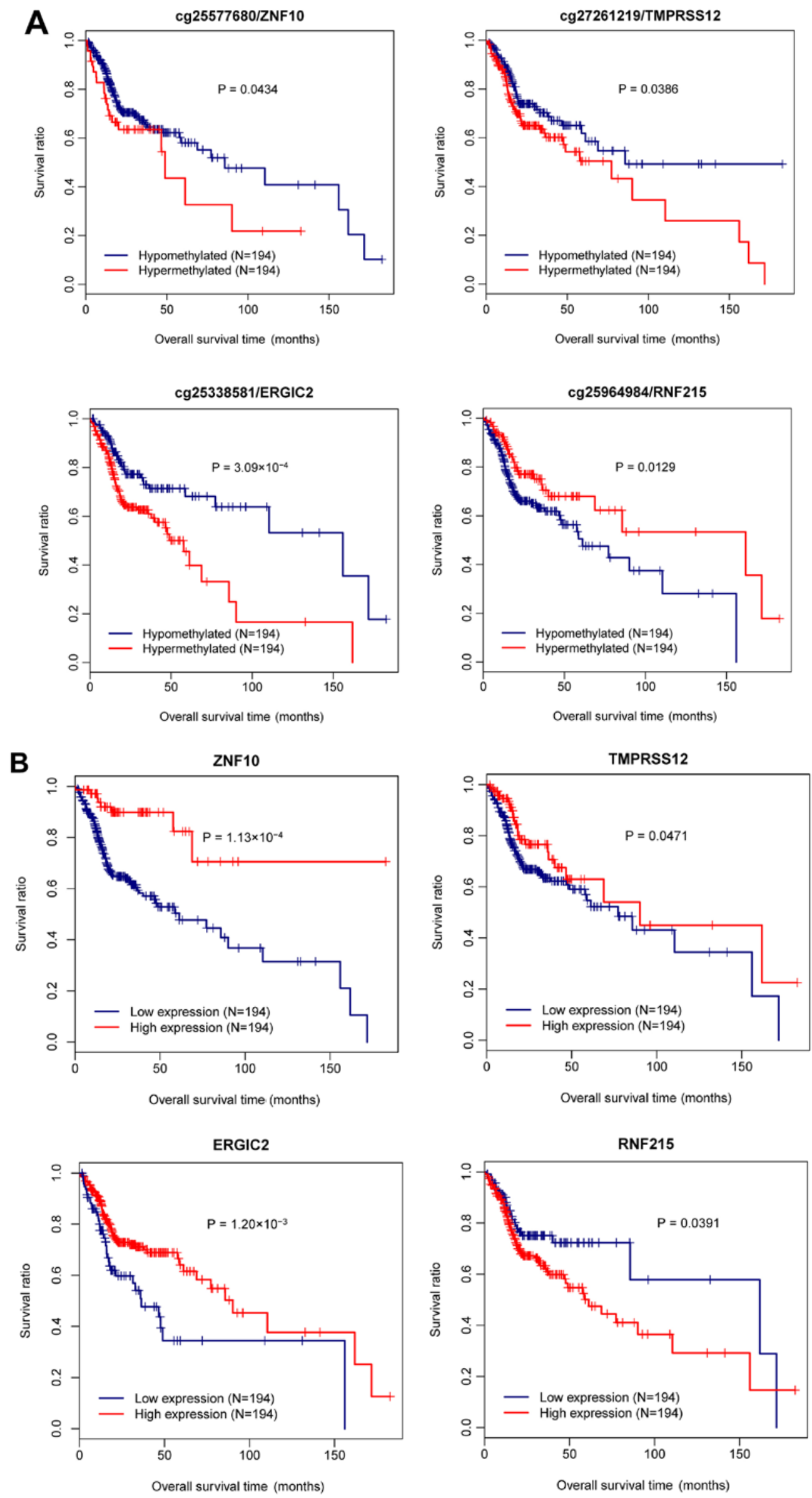

Figure 5. Kaplan-Meier estimates for patients grouped based on (A) median methylation level or (B) median gene expression level of a gene in the training cohort. 
A

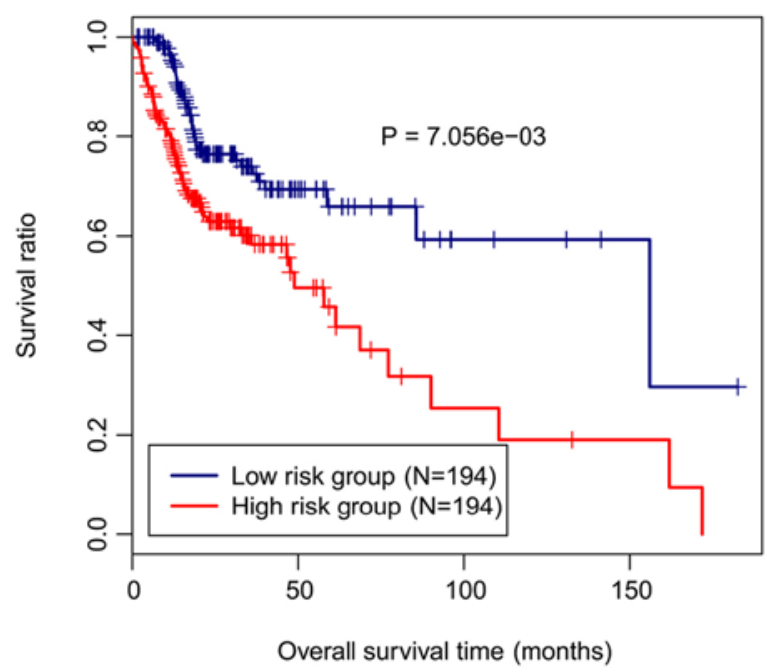

B

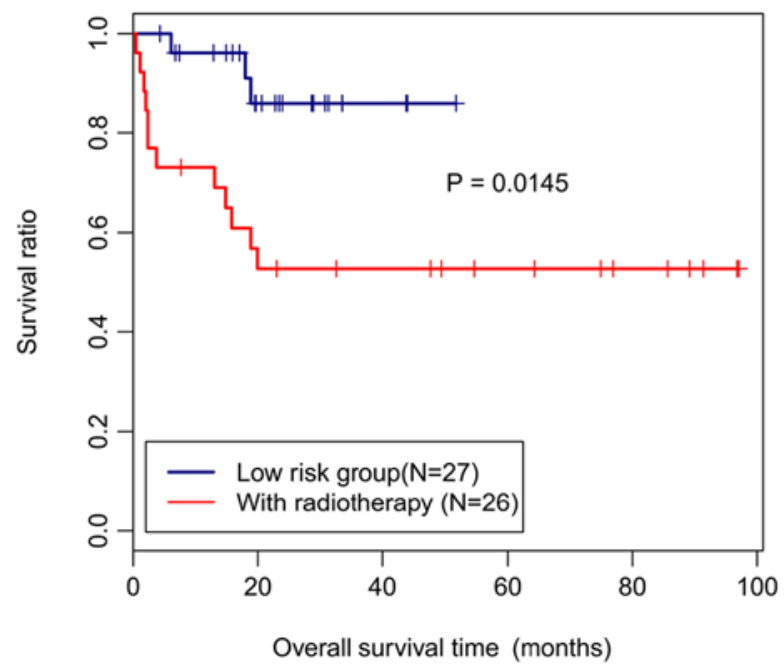

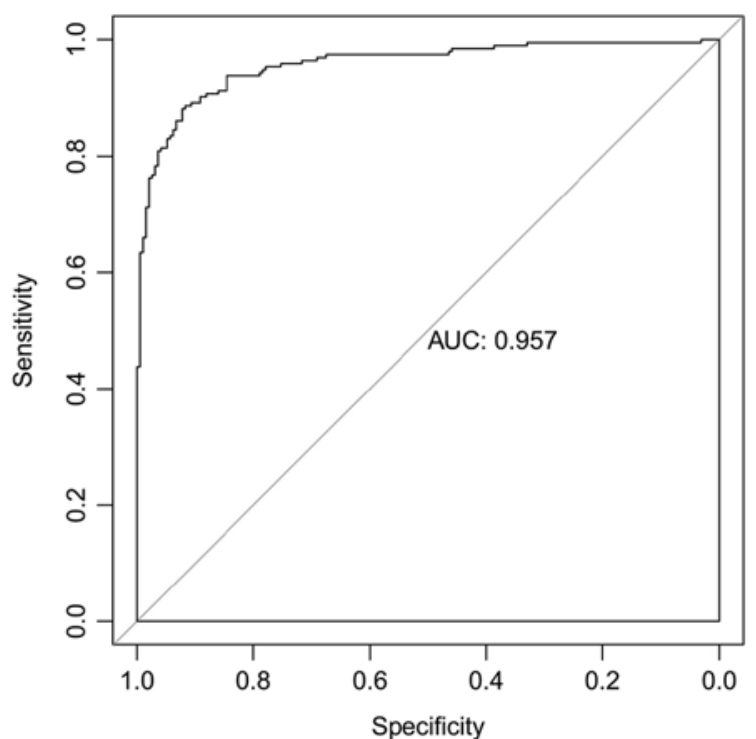

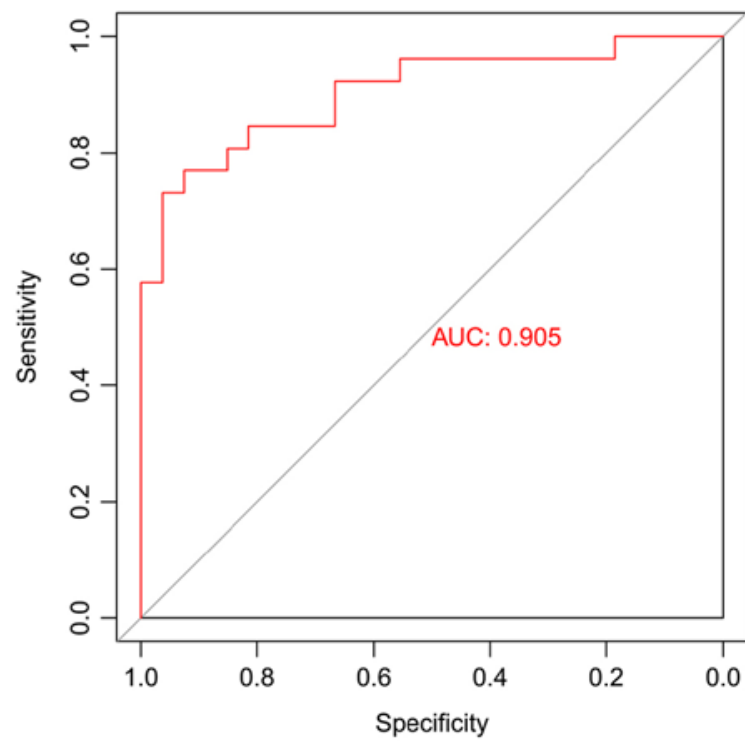

Figure 6. Kaplan-Meier and ROC curves for the four-gene methylation signature in (A) the training set and (B) the validation set. Patients were classified by methylation risk score into high-risk and low-risk groups. Differences between the two groups were evaluated by log-rank test.

A

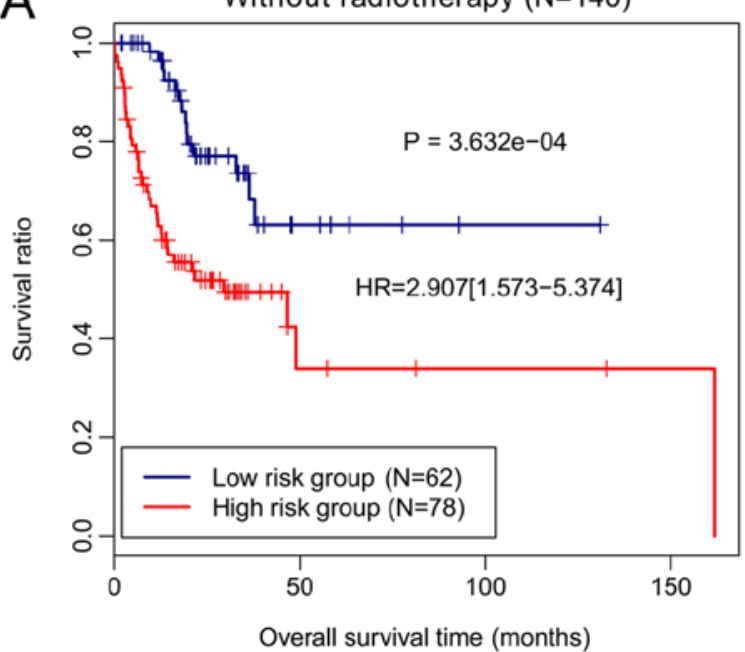

B

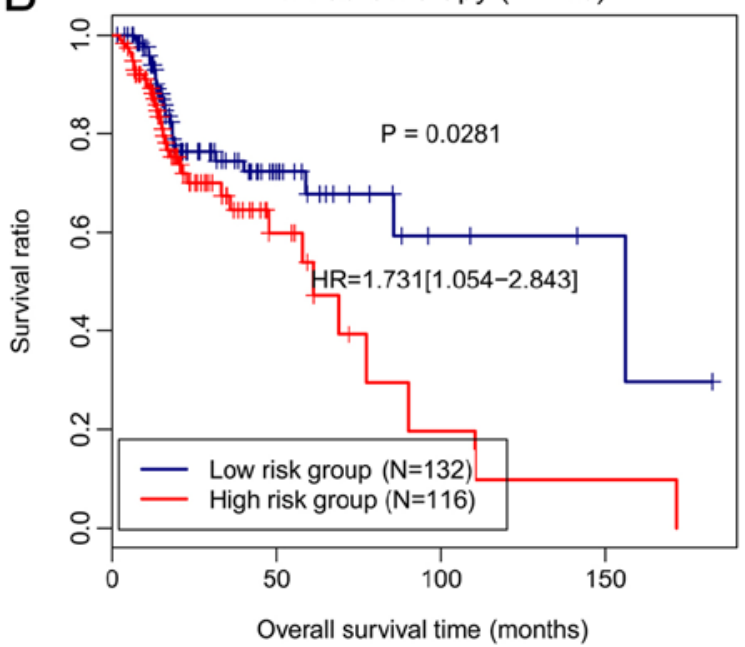

Figure 7. Kaplan-Meier curves for high- and low-risk groups of the patients (A) without or (B) with radiotherapy. 
Table V. Risk score model based on a four-gene methylation signature.

\begin{tabular}{llllllll}
\hline ID & Gene & Chr. & Position & Location & Coef & Hazard ratio (95\% CI) & P-value \\
\hline cg25577680 & ZNF10 & chr12 & 132217652 & Promoter & 0.964 & $6.259(1.274-10.74)$ & 0.0230 \\
cg27261219 & TMPRSS12 & chr12 & 49522905 & TSS & 1.035 & $3.179(1.131-8.935)$ & 0.0277 \\
$\operatorname{cg} 25338581$ & ERGIC2 & chr12 & 29425828 & TSS & 7.166 & $7.576(4.142-10.506)$ & 0.0023 \\
$\operatorname{cg} 25964984$ & RNF215 & chr22 & 29113371 & TSS & -4.896 & $0.437(0.0405-0.719)$ & 0.0223 \\
\hline
\end{tabular}

Chr, chromosome; coef, Cox-PH coefficient.

\section{Discussion}

Despite advancements in the diagnosis and treatment of HNSCC, the prognosis remains poor (24). Radiation therapy is used as a standard adjuvant treatment for HNSCC (25). The present study focused on radiotherapy-related aberrant methylation of genes in HNSCC to identify a prognostic methylation signature. A total of 976 DMGs were revealed between the patients with survival of 12 months or shorter without radiotherapy and those surviving for 48 months or longer and receiving radiotherapy. Moreover, four co-methylation modules that were markedly enriched with DMGs were retrieved by WGCNA analysis. The DMGs in the four modules were functionally associated with protein-DNA complex assembly, nucleosome organization, chromatin assembly, and the cell cycle process. These results may improve our understanding of the mechanisms underlying these DNA methylation alterations in HNSCC.

Following correlation analysis and multivariate Cox regression analysis, a LASSO-penalized Cox-PH model was used to identify the most informative genes for the prediction of survival. LASSO is a popular algorithm with a property of simultaneous variable selection and shrinkage, leading to the identification of prognostic signatures (26). It has been used in Cox-PH model for the survival analysis of patients with breast cancer (27). A four-gene panel predictive of prognosis with the lasso Cox-PH model was established. The risk score derived from the four-gene methylation signature stratified the patients into two risk groups with significantly different OS in both the training and validation sets. ROC curves demonstrated high specificity and sensitivity of the four-gene methylation signature in predicting OS of patients with HNSCC.

The four methylation genes of prognostic value were ZNF10, TMPRSS12, ERGIC2 and RNF215. Zinc finger protein 10 encoded by the $Z N F 10$ gene acts as a transcription repressor and is a member of the zinc finger proteins, the largest transcription factor family involved in development, differentiation, and metabolism. This protein plays versatile roles in cancer progression (28). Chung et al provided evidence that the glioma-associated oncogene family zinc finger 1 is a biomarker in HNSCC (29). However, to the best of our knowledge, the role of ZNF10 in HNSCC is yet unknown. Based on our results, the hypermethylated $\mathrm{CpG}$ sites in $Z N F 10$ were associated with poor prognosis of patients with HNSCC, suggesting that the hypermethylation of ZNF10 may serve as an indicator of poor HNSCC prognosis. The TMPRSS12 gene encodes transmembrane protease, serine 12 , a member of the serine protease family participating in diverse functions such as immune response and blood coagulation and production (30). It has been recognized as an important gene associated with human infertility by genome-wide analyses (31). TMPRSS12 is used as one of the CG signatures in the identification of cancer-associated aberrant DNA methylation that influences gene expression (32). In our study, it had methylated $\mathrm{CpG}$ sites and the hypermethylation was related to poor OS outcome. This observation may suggest that the hypermethylated CpGs in TMPRSS12 may be the causative factor for poor prognosis of HNSCC. ERGIC2, namely $P T X 1$, is a gene determined by subtractive hybridization. The ERGIC2 protein is an endoplasmic reticulum (ER) resident protein implicated in protein trafficking between ER and Golgi bodies (33,34). PTX1 is an alias for the gene PITX1, which has been identified as a predictive indicator of the response to chemotherapy in HNSCC (35). In the present study, as reported for the aforementioned two genes, ERGIC2 also carried methylated $\mathrm{CpG}$ sites, and its hypermethylation was associated with poor survival outcome. Thus, methylated ERGIC2 may be a predictive factor for HNSCC prognosis. The $R N F 125$ gene encodes a novel E3 ubiquitin-protein ligase that may be involved in the T-cell receptor signaling pathway (36). Ring finger ubiquitin protein ligases play a role in tumorigenesis and metastasis (37). Yang et al suggested that RNF125 strengthens p53 degradation and suppresses p53 function (38). The proto-oncoprotein S-phase kinase-associated protein2 ( $\mathrm{Skp} 2$ ) has been revealed to be overexpressed in many malignancies such as HNSCC. Skp2 plays an important role in the degradation of p27 and p21 via the ubiquitin-proteasome pathway (39). In the present study, the hypomethylation and high expression of RNF125 were associated with poor $\mathrm{OS}$, indicative of its function as an oncogene in HNSCC. Further studies are warranted to determine if it functions through the ubiquitin-related pathway.

In the present study, it was revealed that the four-gene methylation signature related to radiotherapy can successfully discriminate between the patients with high risk and low risk. The prognostic ability of this methylation signature was independent of radiotherapy. These findings may improve the risk stratification of patients with HNSCC and is of a particular clinical relevance. Despite these valuable findings, the present study has a few limitations. First, the number of patient samples with available clinical information was small. In addition, these predictive results need to be further validated through substantial experiments.

In conclusion, a radiotherapy-related four-gene methylation signature was identified for predicting HNSCC survival. 
This platform may serve as a guide for the development of individualized therapy for patients with HNSCC. Translation of our findings into future clinical trials requires validation using large populations of patients.

\section{Acknowledgements}

Not applicable.

\section{Funding}

No funding was received.

\section{Availability of data and materials}

The datasets used and/or analyzed during the current study are available from the corresponding author on reasonable request.

\section{Authors' contributions}

JM performed data analyses and wrote the manuscript. RL contributed significantly to data analyses and manuscript revision. JW conceived and designed the study. All authors read and approved the manuscript, and agree to be accountable for all aspects of the research in ensuring that the accuracy or integrity of any part of the work are appropriately investigated and resolved.

\section{Ethics approval and consent to participate}

In the original article of the datasets, the trials were approved by the local institutional review boards of all participating centers, and informed consent was obtained from all patients.

\section{Patient consent for publication}

Not applicable.

\section{Competing interests}

The authors declare that they have no competing interests.

\section{References}

1. GBD 2015 Disease and Injury Incidence and Prevalence Collaborators: Global, regional, and national incidence, prevalence, and years lived with disability for 310 diseases and injuries, 1990-2015: A systematic analysis for the Global Burden of Disease Study 2015. Lancet 388: 1545-1602, 2016.

2. Leemans CR, Snijders PJF and Brakenhoff RH: The molecular landscape of head and neck cancer. Nat Rev Cancer 18: 269-282, 2018.

3. López-Verdín S, Lavalle-Carrasco J, Carreón-Burciaga RG, Serafín-Higuera N, Molina-Frechero N, González-González R and Bologna-Molina R: Molecular markers of anticancer drug resistance in head and neck squamous cell carcinoma: A literature review. Cancers (Basel) 10: E376, 2018.

4. Beyzadeoglu M, Selek U and Ozyigit G (eds): Radiation Therapy for Head and Neck Cancers: A Case-Based Review. Springer, New York, NY, pp244, 2014.

5. Delpu Y, Cordelier P, Cho WC and Torrisani J: DNA methylation and cancer diagnosis. Int J Mol Sci 14: 15029-15058, 2013.

6. Klutstein M, Nejman D, Greenfield R and Cedar H: DNA methylation in cancer and aging. Cancer Res 76: 3446-3450, 2016.
7. Lechner M, Fenton T, West J, Wilson G, Feber A, Henderson S, Thirlwell C, Dibra HK, Jay A, Butcher L, et al: Identification and functional validation of HPV-mediated hypermethylation in head and neck squamous cell carcinoma. Genome Med 5: 15, 2013.

8. Steinmann K, Sandner A, Schagdarsurengin U and Dammann RH: Frequent promoter hypermethylation of tumor-related genes in head and neck squamous cell carcinoma. Oncol Rep 22: 1519-1526, 2009.

9. Kostareli E, Hielscher T, Zucknick M, Baboci L, Wichmann G, Holzinger D, Mücke O, Pawlita M, Del Mistro A, BoscoloRizzo P, et al: Gene promoter methylation signature predicts survival of head and neck squamous cell carcinoma patients. Epigenetics 11: 61-73, 2016.

10. Kostareli E, Holzinger D, Bogatyrova O, Hielscher T, Wichmann G, Keck M, Lahrmann B, Grabe N, Flechtenmacher C, Schmidt CR, et al: HPV-related methylation signature predicts survival in oropharyngeal squamous cell carcinomas. J Clin Invest 123: 2488-2501, 2013.

11. Esposti DD, Sklias A, Lima SC, Beghelli-de la Forest Divonne S, Cahais V, Fernandez-Jimenez N, Cros MP, Ecsedi S, Cuenin C, Bouaoun L, et al: Unique DNA methylation signature in HPV-positive head and neck squamous cell carcinomas. Genome Med 9: 33, 2017.

12. Shuryak I, Hall EJ and Brenner DJ: Dose dependence of accelerated repopulation in head and neck cancer: Supporting evidence and clinical implications. Radiother Oncol 127: 20-26, 2018.

13. Zhu X, Wang Y, Tan L and Fu X: The pivotal role of DNA methylation in the radio-sensitivity of tumor radiotherapy. Cancer Med 7: 3812-3819, 2018.

14. Huang KH, Huang SF, Chen IH, Liao CT, Wang HM and Hsieh LL: Methylation of RASSF1A, RASSF2A, and HIN-1 is associated with poor outcome after radiotherapy, but not surgery, in oral squamous cell carcinoma. Clin Cancer Res 15: 4174-4180, 2009

15. Krishnan NM, Dhas K, Nair J, Palve V, Bagwan J, Siddappa G, Suresh A, Kekatpure VD, Kuriakose MA and Panda B: A minimal DNA methylation signature in oral tongue squamous cell carcinoma links altered methylation with tumor attributes. Mol Cancer Res 14: 805-819, 2016.

16. Ritchie ME, Phipson B, Wu D, Hu Y, Law CW, Shi W and Smyth GK: Limma powers differential expression analyses for RNA-sequencing and microarray studies. Nucleic Acids Res 43: e47, 2015.

17. Langfelder $P$ and Horvath S: WGCNA: An R package for weighted correlation network analysis. BMC Bioinformatics 9: $559,2008$.

18. Massart R, Dymov S, Millecamps M, Suderman M, Gregoire S, Koenigs K, Alvarado S, Tajerian M, Stone LS and Szyf M: Overlapping signatures of chronic pain in the DNA methylation landscape of prefrontal cortex and peripheral T cells. Sci Rep 6: 19615, 2016.

19. Cao J and Zhang S: A bayesian extension of the hypergeometric test for functional enrichment analysis. Biometrics 70: 84-94, 2014

20. Gene Ontology Consortium: Gene ontology consortium: Going forward. Nucleic Acids Res 43: D1049-D1056, 2015.

21. Huang DW, Sherman BT, Tan Q, Kir J, Liu D, Bryant D, Guo Y, Stephens R, Baseler MW, Lane HC and Lempicki RA: DAVID Bioinformatics Resources: Expanded annotation database and novel algorithms to better extract biology from large gene lists. Nucleic Acids Res 35: W169-W175, 2007.

22. Tibshirani R: The lasso method for variable selection in the Cox model. Stat Med 16: 385-395, 1997.

23. Goel MK, Khanna P and Kishore J: Understanding survival analysis: Kaplan-Meier estimate. Int J Ayurveda Res 1: 274-278, 2010.

24. Marur S and Forastiere AA: Head and neck squamous cell carcinoma: Update on epidemiology, diagnosis, and treatment. Mayo Clin Proc 91: 386-396, 2016.

25. Marur S and Forastiere AA: Head and neck cancer: Changing epidemiology, diagnosis, and treatment. Mayo Clin Proc 83: 489-501, 2008.

26. Bøvelstad HM, Nygård S, Størvold HL, Aldrin M, Borgan $\varnothing$, Frigessi A and Lingjaerde OC: Predicting survival from microarray data-a comparative study. Bioinformatics 23: 2080-2087, 2007.

27. Goeman JJ: L1 penalized estimation in the Cox proportional hazards model. Biom J 52: 70-84, 2010.

28. Jen $\mathrm{J}$ and Wang YC: Zinc finger proteins in cancer progression. J Biom Sci 23: 53, 2016. 
29. Chung CH, Dignam JJ, Hammond ME, Klimowicz AC, Petrillo SK Magliocco A, Jordan R, Trotti A, Spencer S, Cooper JS, et al: Glioma-associated oncogene family zinc finger 1 expression and metastasis in patients with head and neck squamous cell carcinoma treated with radiation therapy (RTOG 9003). J Clin Oncol 29: 1326-1334, 2011.

30. Hedstrom L: Serine protease mechanism and specificity. Chem Rev 34: 4501-4524, 2003.

31. Liu M, Hu Z, Qi L, Wang J, Zhou T, Guo Y, Zeng Y, Zheng B, Wu Y, Zhang $\mathrm{P}$, et al: Scanning of novel cancer/testis proteins by human testis proteomic analysis. Proteomics 13: 1200-1210, 2013.

32. Saghafinia S, Mina M, Riggi N, Hanahan D and Ciriello G: Pan-cancer landscape of aberrant DNA methylation across human tumors. Cell Rep 25: 1066-1080.e8, 2018.

33. Kwok SC, Liu X, Mangel P and Daskal I: PTX1(ERGIC2)-VP22 fusion protein upregulates interferon-beta in prostate cancer cell line PC-3. DNA Cell Biol 25: 523-529, 2006.

34. Kwok SC, Kumar S and Dai G: Characterization of a variant of ERGIC2 transcript. DNA Cell Biol 33: 73-78, 2014.

35. Takenobu M, Osaki M, Fujiwara K, Fukuhara T, Kitano H, Kugoh H and Okada F: PITX1 is a novel predictor of the response to chemotherapy in head and neck squamous cell carcinoma. Mol Clin Oncol 5: 89-94, 2016.
36. Chu P, Pardo J, Zhao H, Li CC, Pali E, Shen MM, Qu K, Yu SX, Huang BC, Yu P, et al: Systematic identification of regulatory proteins critical for T-cell activation. J Biol 2: 21, 2003.

37. Fang S, Lorick KL, Jensen JP and Weissman AM: RING finger ubiquitin protein ligases: Implications for tumorigenesis, metastasis and for molecular targets in cancer. Semin Cancer Biol 13: 5-14, 2003.

38. Yang L, Zhou B, Li X, Lu Z, Li W, Huo X and Miao Z: RNF125 is a ubiquitin-protein ligase that promotes p53 degradation. Cell Physiol Biochem 35: 237-245, 2015.

39. Khan AQ, Siveen KS,Prabhu KS, Kuttikrishnan S, Akhtar S, Shaar A, Raza A, Mraiche F, Dermime S and Uddin S: Curcumin-mediated degradation of s-phase kinase protein 2 induces cytotoxic effects in human papillomavirus-positive and negative squamous carcinoma cells. Front Onco 8: 399, 2018.

This work is licensed under a Creative Commons Attribution-NonCommercial-NoDerivatives 4.0 International (CC BY-NC-ND 4.0) License. 